\title{
REGENERAÇÃo NATURAL EM ÁREAS DESMATADAS E DOMINADAS POR Pteridium aquilinum (L.) Kuhn. NA SERRA DA MANTIQUEIRA
}

\author{
Selma Cristina Ribeiro' ${ }^{1}$, Soraya Alvarenga Botelho' ${ }^{2}$ Marco Aurélio Leite Fontes ${ }^{3}$, \\ Paulo Oswaldo Garcia ${ }^{4}$, Hisaías de Souza Almeida ${ }^{5}$
}

(recebido: 9 de julho de 2010; aceito: 28 de setembro de 2012)

\begin{abstract}
RESUMO: Objetivou-se analisar a sucessão ecológica em áreas desmatadas e dominadas espontaneamente por Pteridium aquilinum na Serra da Mantiqueira, por meio do levantamento da regeneração natural de espécies arbustivo-arbóreas, bem como avaliar a influência dos históricos de perturbação e condições edáficas. Amostraram-se duas áreas em Bocaina de Minas, Minas Gerais, em regeneração há 6 e 20 anos, com 60 parcelas de $10 \mathrm{~m}^{2}$, inventariando-se os regenerantes de 0,15 a $3 \mathrm{~m}$ de altura, todos identificados e medidos em diâmetro e altura. Registraram-se 1.159 indivíduos de 53 espécies, destacando-se a família Melastomataceae pela maior riqueza e abundância. As áreas diferenciaram-se na composição de espécies, com similaridade de Jaccard de 3,7\%. Sob análise de correspondência canônica, a área de seis anos evidenciou correlações positivas entre a comunidade regenerante e teores de fósforo não lábil e argila, enquanto a área de 20 anos mostrou correlações com K, P, Ca, Al, pH e soma de bases trocáveis, além de maiores densidades de Pteridium. Os resultados demonstraram que a dominância de P. aquilinum conduz a uma sucessão ecológica sob inibição, onde a samambaia atua negativamente sobre a riqueza e abundância de populações arbustivo-arbóreas. Também se confirmou a preferência da espécie por terrenos declivosos, a maiores altitudes, com solos mais ácidos, beneficiando-se também de distúrbios. Por outro lado, chama-se a atenção para a suscetibilidade desses ambientes montanos e para a importância da preservação de sua vegetação natural, assim como do desbaste das samambaias como estratégia de restauração florestal.
\end{abstract}

Palavras-chave: Sucessão ecológica, dinâmica florestal, florestas montanas, restauração ecológica, resiliência ecológica.

\section{NATURAL REGENERATION OF DEFORESTED AREAS DOMINATED BY Pteridium aquilinum (L.) Kuhn LOCATED IN THE SERRA DA MANTIQUEIRA MOUNTAIN RANGE}

\begin{abstract}
This study was set out with the objective of analyzing successional process in areas which are deforested and dominated by Pteridium aquilinum in the Serra da Mantiqueira mountain range, by researching the natural regeneration of shrub and tree species and evaluating both disturbance history and the edaphic conditions on the natural regeneration community. This research investigated two abandoned pasture areas in Bocaina de Minas county exposed to natural regeneration intervals ranging from six years (area named 6A) to twenty years (area named 20A). The inventory occurred from sixty plots of $10 \mathrm{~m}^{2}$, where all samples surveyed were between $0.15 \mathrm{~m}$ and $3 \mathrm{~m}$ high. All samples were identified and both the diameter in ground level and total height of the specimens were measured. The survey totaled 1,159 samples and 53 species. Melastomataceae was registered with the highest species richness and the highest specimen abundance. The two sampled areas showed species composition differences, with Jaccard similarity coefficient equal to 3.7\%. The canonical correspondence analysis showed the correlations between natural regeneration stratum and non-labile phosphorus and clay in the $6 \mathrm{~A}$ area. On the other hand, the 20A area showed correlations between plant regeneration and the $\mathrm{K}, \mathrm{P}, \mathrm{Ca}^{2+}, \mathrm{Al}^{3+}$ levels, with higher $\mathrm{pH}$ levels, and with the sum of exchangeable bases. In addition, the vegetation surveyed in area $20 A$ was correlated with higher Pteridium population density. The results showed that the dominance of Pteridium aquilinum leads to successional process under inhibition, in which the ferns act negatively on the richness and abundance of shrub populations. It was also confirmed the Pteridium's affinity to steep areas, mainly in higher altitudes, where the soil is acid, as well as its preference to disturbed areas. Moreover, we highlight the fragilities of the mountain environments and the importance of preserving natural vegetation, as well as the bracken management as a strategy for forest restoration.
\end{abstract}

Key words: Successional process, forest dynamic, mountain forests, ecological restoration, ecological resilience.

\footnotetext{
'Engenheira Florestal, Mestre em Ciências Florestais - Instituto Chico Mendes de Conservação da Biodiversidade - Área de Proteção Ambiental Serra da Mantiqueira - Rodovia BR 354, km 48 - Horto Florestal - 37466-000 - Itamonte, MG, Brasil selribeiro2@yahoo.com.br

${ }^{2}$ Engenheira Florestal, Professora Doutora em Engenharia Florestal - Universidade Federal de Lavras/UFLA - Departamento de Ciências Florestais - Cx. P. 3037 - 37200-000 - Lavras, MG, Brasil - soraya@dcf.ufla.br

${ }^{3}$ Engenheiro Florestal, Professor Doutor em Ecologia - Universidade Federal de Lavras/UFLA - Departamento de Ciências Florestais Cx. P. 3037 - 37200-000 - Lavras, MG, Brasil -fontes@dcf.ufla.br

${ }^{4}$ Biólogo, Professor Doutor em Engenharia Florestal - Universidade Federal de Lavras/UFLA - Departamento de Ciências Florestais Cx. P. 3037 - 37200-000 - Lavras, MG, Brasil - paulogarciamg@ posgrad.ufla.br

${ }^{5}$ Biólogo, Doutor em Engenharia Florestal - Universidade Federal de Alfenas - Instituto de Ciências da Natureza - Rua Gabriel Monteiro da Silva, 700 - 37130-000 - Alfenas, MG, Brasil - hisaias37@gmail.com
}

Cerne, Lavras, v. 19, n. 1, p. 65-76, jan./mar. 2013 


\section{INTRODUÇÃO}

A Mata Atlântica constitui um dos ecossistemas mais ameaçados (FUNDAÇÃO SOS MATA ATLÂNTICA; INSTITUTO NACIONAL DE PESQUISAS ESPACIAIS INPE, 2009), com seus principais remanescentes em áreas de relevo acidentado, como as serras do Mar e da Mantiqueira (CAPOBIANCO, 2001). No sul de Minas Gerais, a Mata Atlântica é representada por pequenos fragmentos florestais, menores que 10 ha (OLIVEIRA-FILHO et al., 1997), de diferentes estádios sucessionais (SOUZA et al., 2002). A dinâmica de clareira iniciada com as perturbações (BROKAW, 1982; DENSLOW, 1980) é relacionada à frequência e severidade dos distúrbios (CONNELL, 1978), à permanência do banco de sementes ou propágulos (HONDA, 2008; RODRIGUES et al., 2004) e à proximidade de fontes de novos propágulos (FRELICH et al., 1998). Esse processo geralmente contribui para a manutenção da biodiversidade, ao prevenir a exclusão competitiva (CONNELL, 1978). Ao contrário, porém, a sucessão ecológica pode seguir um modelo de "inibição", quando o distúrbio favorece a poucas espécies (CONNELL; SLATYER, 1977).

A regeneração das comunidades arbóreas no domínio Atlântico ocorre, com frequência, simultaneamente à colonização por densas populações de Pteridium aquilinum (L.) Kuhn., que retarda a regeneração florestal (SILVA; MATOS, 2006) e compromete a conexão entre os fragmentos (PINHEIRO; DURIGAN, 2009; RONDONNETO et al., 2000). Nesse contexto, os efeitos negativos de $P$. aquilinum potencializam-se com a falta de informações sobre suas interações ecológicas, importantes na recuperação de áreas degradadas e dominadas pela espécie.

O objetivo, neste trabalho, foi analisar a sucessão ecológica em áreas desmatadas e dominadas espontaneamente por $P$. aquilinum na Serra da Mantiqueira, por meio do levantamento da regeneração natural de suas espécies arbustivo-arbóreas, bem como avaliar a influência das diferenças nos históricos de perturbação e condições edáficas.

\section{MATERIAL E MÉTODOS}

O trabalho ocorreu nas proximidades da cabeceira do rio Grande, em Bocaina de Minas, sul de Minas Gerais, na Área de Proteção Ambiental da Serra da Mantiqueira, de clima Cb de Köppen (subtropical úmido e mesotérmico), com médias anuais de temperatura e precipitação respectivas de $16,7^{\circ} \mathrm{C}$ e $2.108 \mathrm{~mm}$ (CARVALHO et al., 2005) e predomínio de florestas ombrófilas, onde selecionaram- se duas áreas para inventário da regeneração arbustivoarbórea. A primeira, insere-se na Reserva Particular do Patrimônio Natural Alto Rio Grande (UTM 23K 544064; 7541825; Datum SAD69), possui 2 ha dominados por Pteridium aquilinum (doravante Pteridium), cercados por floresta, e se encontra em regeneração natural há 20 anos (área 20A), desde o abandono da pastagem formada por desmate e cultivo de Melinis minutiflora P. Beauv. A segunda (a $14 \mathrm{~km}$; 23K 555599; 7549864), possui 1,5 ha dominados por Pteridium, cercados por floresta, campo natural e nascente, em regeneração há 6 anos (área 6A), desde o abandono da pastagem de gramíneas nativas como Andropogon leucostachyus Kunth.

Em cada área, alocaram-se 30 parcelas de $2 \times 5 \mathrm{~m}$ (total de $600 \mathrm{~m}^{2}$ ), distanciadas em $5 \mathrm{~m}$ e distribuídas em transeções (equidistantes $10 \mathrm{~m}$ ) no sentido do maior declive. Em cada parcela, registraram-se os indivíduos arbustivo-arbóreos com altura entre 0,15 e $3 \mathrm{~m}$, medidos em altura e diâmetro ao nível do solo, e coletado material botânico para identificação em herbário ou por consulta à literatura e especialistas. Adotou-se o sistema de classificação do Angiosperm Phylogeny Group - APG (2003). Calcularam-se, por espécie, a densidade, frequência e dominância, absolutos e relativos (MUELLERDOMBOIS; ELLEMBERG, 1974) e, para a comunidade, a equabilidade de Pielou e o índice de diversidade de Shannon (KREBS, 1989), este último comparado entre as áreas pelo teste " $t$ " de Hutchenson (ZAR, 1996). Foi mensurada a cobertura percentual (BRAUN-BLANQUET, 1979) de Pteridium por parcela e sua altura, cujas diferenças foram verificadas pelo teste "t" de Student (ZAR, 1996).

Nas áreas, predominam Cambissolos Háplicos, geralmente cascalhentos, rasos, pouco desenvolvidos e de permeabilidade mediana (EMPRESA BRASILEIRA DE PESQUISA AGROPECUÁRIA - EMBRAPA, 2006). Para detalhamento, no centro de cada parcela foram coletadas amostras de solo superficial ( 0 a $20 \mathrm{~cm}$ ), submetidas a análises química e textural no Laboratório de Análise de Solos da UFLA: $\mathrm{pH}$, teores de $\mathrm{P}, \mathrm{K}, \mathrm{Ca}^{2+}, \mathrm{Mg}^{2+}, \mathrm{Al}^{3+}, \mathrm{H}+\mathrm{Al}$, soma de bases trocáveis (SB), capacidade de troca catiônica (CTC), CTC efetiva (t), CTC a pH 7 (T), saturação por bases (V) e alumínio (m), matéria orgânica (MO), fósforo não-lábil ou remanescente (P-rem), teores de areia, silte e argila. Para cada área, realizaram-se análises dos parâmetros edáficos absolutos, a fim de verificar possíveis variações por localidade. Com o uso do software BioEstat 5 (AYRES, 2005) foram realizados tanto o teste " $\mathrm{t}$ " de Student como as ANOVAunifatoriais associadas a testes de Tukey (ZAR, 1996).

Cerne, Lavras, v. 19, n. 1, p. 65-76, jan./mar. 2013 
As variáveis de solo, topográficas e populacionais de Pteridium obtidas por parcela foram usadas em análise de correspondência canônica (CCA) (BRAAK, 1987) para verificar gradientes na comunidade de regenerantes correlacionados às variáveis mensuradas, com uso do softwarePC-Ord 5.1 (MCCUNE; MEFFORD, 2006). A existência de colinearidade entre variáveis foram verificadas, visto que a redundância entre as mesmas pode afetar a ordenação (HAIR JÚNIOR et al., 2009). Relações florísticas entre as áreas foram verificadas pelo coeficiente de similaridade de Jaccard (MUELLER-DOMBOIS;ELLEMBERG 1974).

\section{RESULTADOS E DISCUSSÃO}

Foram amostrados 1.159 regenerantes, distribuídos em 53 espécies, 36 gêneros e 21 famílias (Tabela 1). Aárea 20A apresentou 218 indivíduos (18,8\% do total) e 21 espécies, enquanto em 6A foram 941 indivíduos $(81,2 \%)$ e 34 espécies (Tabela 1). De modo similar, porém no domínio de Cerrado, Miatto et al. (2011) encontraram até 28 espécies arbóreas em trechos colonizados por Pteridium. No entanto, o presente estudo focou o estrato regenerativo, onde as espécies podem experimentar acentuadas taxas de mortalidade (UHL et al., 1988), o que empobrece a comunidade e dificulta inferências sobre estágios posteriores da sucessão e sobre a riqueza registrada.
Melastomataceae se destacou pela abundância (65,7\% dos indivíduos) em ambas as áreas, seguida por Euphorbiaceae $(18,1 \%)$, sendo que essas famílias totalizaram 972 indivíduos. Melastomataceae apresentou a maior riqueza de espécies (13 espécies). Nos fragmentos florestais da região do alto rio Grande é reconhecida a representatividade de Melastomataceae na estrutura e riqueza (CARVALHO et al., 2005; OLIVEIRA-FILHO, et al., 2004; PINTO et al., 2005; RONDON NETO et al., 2000), aumentando em importância em áreas de maior altitude (CARVALHO et al., 2005; OLIVEIRA-FILHO et al., 2004; OLIVEIRA-FILHO; FONTES, 2000), como observado nas áreas 20A e 6A (altitudes superiores a $1.100 \mathrm{~m}$ ). Silva e Silva-Matos (2006) também registraram Melastomataceae dentre as principais famílias na regeneração natural em áreas colonizadas por $P$. aquilinum, em florestas ombrófilas no Rio de Janeiro. Assim, os resultados obtidos no presente estudo não se relacionam apenas aos aspectos fitogeográficos. As espécies de Melastomataceae caracterizam-se pela grande produção de sementes, em geral de tamanhos reduzidos (JOLY, 1998). Pressupõe-se que isso incrementaria a probabilidade das sementes transporem a densa serapilheira formada pelas frondes da samambaia, atingindo o solo, o que repercutiria no destaque da família.

Tabela 1 - Parâmetros estruturais das espécies arbustivas e arbóreas inventariadas na regeneração natural de duas áreas colonizadas por Pteridium aquilinum em Bocaina de Minas, MG. Onde: N: número total de indivíduos; DA: densidade absoluta; DR: densidade relativa; DoA: dominância absoluta; DoR: dominância relativa; FA: frequência absoluta, FR: frequência relativa; VI: valor de importância.

Table 1 - Shrub and tree species structural parameters registered in natural regeneration inventory in two areas colonized by Pteridium aquilinum, both located in Bocaina de Minas, MG. N: total number of samples; DA, absolute density; DR, relative density; DoA, absolute dominance; DoR, relative dominance; FA, absolute frequency; FR, relative frequency; VI, importance value.

\begin{tabular}{|c|c|c|c|c|c|c|c|c|}
\hline Área 20A & $\mathrm{N}$ & $\mathrm{DA}(\mathrm{n} / \mathrm{ha})$ & $\mathrm{DR}(\%)$ & DoA $\left(\mathrm{m}^{2} / \mathrm{ha}\right)$ & $\operatorname{DoR}(\%)$ & FA $(\%)$ & FR $(\%)$ & $\mathrm{VI}(\%)$ \\
\hline \multicolumn{9}{|l|}{ Asteraceae } \\
\hline Eremanthus erythropappus (DC.) MacLeish & 8 & 266,6 & 3,67 & 0,003 & 0,05 & 4,55 & 1,15 & 4,87 \\
\hline Wulffia stenoglossa (L.) DC. & 1 & 33,3 & 0,46 & 0,001 & 0,02 & 4,55 & 1,15 & 1,63 \\
\hline \multicolumn{9}{|l|}{ Bignoniaceae } \\
\hline Handroanthus ochraceus (Cham.) Mattos & 7 & 233,3 & 3,21 & 0,13 & 2,39 & 18,18 & 4,60 & 10,20 \\
\hline Jacaranda macrantha Cham. & 2 & 66,6 & 0,92 & 0,04 & 0,75 & 9,09 & 2,30 & 3,97 \\
\hline \multicolumn{9}{|l|}{ Clethraceae } \\
\hline Clethra scabra Pers. & 10 & 333,3 & 4,59 & 0,09 & 1,63 & 40,91 & 10,34 & 16,56 \\
\hline \multicolumn{9}{|l|}{ Euphorbiaceae } \\
\hline Alchornea glandulosa Poepp. \& Endl. & 3 & 100 & 1,38 & 0,01 & 0,14 & 9,09 & 2,30 & 3,82 \\
\hline
\end{tabular}


Tabela 1 - Continuação...

Table 1 - Continued...

\begin{tabular}{|c|c|c|c|c|c|c|c|c|}
\hline Área 20A & $\mathrm{N}$ & DA (n/ha) & $\mathrm{DR}(\%)$ & $\operatorname{DoA}\left(\mathrm{m}^{2} / \mathrm{ha}\right)$ & $\operatorname{DoR}(\%)$ & FA $(\%)$ & FR $(\%)$ & $\mathrm{VI}(\%)$ \\
\hline \multicolumn{9}{|l|}{ Melastomataceae } \\
\hline Leandra scabra DC. & 54 & 1.800 & 24,77 & 0,18 & 3,18 & 54,55 & 13,79 & 41,74 \\
\hline Bellucia sp. & 43 & $1.433,3$ & 19,72 & 4,23 & 76,03 & 40,91 & 10,34 & 106,10 \\
\hline Miconia latecrenata (DC.) Naudin & 25 & 833,3 & 11,47 & 0,05 & 0,87 & 40,91 & 10,34 & 22,68 \\
\hline Miconia mellina $\mathrm{DC}$ & 10 & 333,3 & 4,59 & 0,24 & 4,25 & 27,27 & 6,90 & 15,73 \\
\hline Miconia calvescens Schrank \& Mart. ex DC. & 9 & 300 & 4,13 & 0,24 & 4,24 & 31,82 & 8,05 & 16,41 \\
\hline Miconia sellowiana Naudin & 4 & 133,3 & 1,83 & 0,05 & 0,86 & 13,64 & 3,45 & 6,14 \\
\hline Tibouchina estrellensis (Raddi) Cogn. & 3 & 100 & 1,38 & 0,06 & 1,00 & 9,09 & 2,30 & 4,67 \\
\hline \multicolumn{9}{|l|}{ Meliaceae } \\
\hline Cedrela fissilis Vell. & 1 & 33,3 & 0,46 & 0,09 & 1,59 & 4,55 & 1,15 & 3,20 \\
\hline \multicolumn{9}{|l|}{ Myrsinaceae } \\
\hline Myrsine umbellata Mart. & 9 & 300 & 4,13 & 0,08 & 1,50 & 22,73 & 5,75 & 11,37 \\
\hline \multicolumn{9}{|l|}{ Myrtaceae } \\
\hline Marlierea laevigata (DC.) Kiaersk. & 1 & 33,3 & 0,46 & 0,002 & 0,03 & 4,55 & 1,15 & 1,64 \\
\hline Myrcia splendens (Sw.) DC. & 1 & 33,3 & 0,46 & 0,03 & 0,51 & 4,55 & 1,15 & 2,12 \\
\hline \multicolumn{9}{|l|}{ Rubiaceae } \\
\hline Psychotria vellosiana Benth. & 22 & 733,3 & 10,09 & 0,03 & 0,49 & 36,36 & 9,20 & 19,78 \\
\hline Amaioua guianensis Aubl. & 1 & 33,3 & 0,46 & 0,0009 & 0,02 & 4,55 & 1,15 & 1,63 \\
\hline \multicolumn{9}{|l|}{ Salicaceae } \\
\hline Casearia decandra Jacq. & 1 & 33,3 & 0,46 & 0,0002 & 0,003 & 4,55 & 1,15 & 1,61 \\
\hline \multicolumn{9}{|l|}{ Thymelaeaceae } \\
\hline Daphnopsis fasciculata (Meisn.) Nevling & 3 & 100 & 1,38 & 0,03 & 0,45 & 9,09 & 2,30 & 4,13 \\
\hline Total & 218 & $7.266,6$ & 100 & 5,56 & 100 & 395,45 & 100 & 300 \\
\hline Área 6A & $\mathrm{N}$ & DA (n/ha) & $\operatorname{DR}(\%)$ & $\operatorname{DoA}\left(\mathrm{m}^{2} / \mathrm{ha}\right)$ & $\operatorname{DoR}(\%)$ & FA $(\%)$ & FR $(\%)$ & $\mathrm{VI}(\%)$ \\
\hline \multicolumn{9}{|l|}{ Aquifoliaceae } \\
\hline Ilex brevicuspis Reissek & 1 & 66,6 & 0,21 & 0,01 & 0,01 & 3,33 & 0,61 & 0,83 \\
\hline \multicolumn{9}{|l|}{ Asteraceae } \\
\hline Asteraceae sp.1 & 1 & 33,3 & 0,11 & 0,001 & 0,001 & 3,33 & 0,61 & 0,72 \\
\hline Gochnatia polymorpha (Less.) Cabrera & 1 & 33,3 & 0,11 & 0,01 & 0,01 & 3,33 & 0,61 & 0,73 \\
\hline Piptocarpha axillaris (Less.) Baker & 2 & 66,6 & 0,21 & 0,20 & 0,18 & 6,67 & 1,22 & 1,61 \\
\hline Vernonanthura divaricata (Spreng.) H. Rob. & 1 & 33,3 & 0,11 & 0,01 & 0,01 & 3,33 & 0,61 & 0,73 \\
\hline \multicolumn{9}{|l|}{ Bignoniaceae } \\
\hline Jacaranda puberula Cham. & 4 & 133,3 & 0,42 & 0,03 & 0,03 & 10,00 & 1,83 & 2,28 \\
\hline \multicolumn{9}{|l|}{ Euphorbiaceae } \\
\hline Croton floribundus Spreng. & 208 & 6933,3 & 22,08 & 0,05 & 0,04 & 36,67 & 6,71 & 28,83 \\
\hline Sapium glandulosum (L.) Morong & 2 & 66,6 & 0,21 & 0,004 & 0,004 & 3,33 & 0,61 & 0,83 \\
\hline
\end{tabular}


Tabela 1 - Continuação...

Table 1 - Continued...

\begin{tabular}{|c|c|c|c|c|c|c|c|c|}
\hline Área 6A & $\mathrm{N}$ & DA (n/ha) & $\mathrm{DR}(\%)$ & $\operatorname{DoA}\left(\mathrm{m}^{2} / \mathrm{ha}\right)$ & $\operatorname{DoR}(\%)$ & FA $(\%)$ & FR $(\%)$ & $\mathrm{VI}(\%)$ \\
\hline \multicolumn{9}{|l|}{ Fabaceae } \\
\hline Apuleia leiocarpa (Vogel) J.F.Macbr. & 1 & 33,3 & 0,11 & 0,23 & 0,21 & 3,33 & 0,61 & 0,93 \\
\hline Dalbergia frutescens (Vell.) Britton & 5 & 166,6 & 0,53 & 0,002 & 0,002 & 16,67 & 3,05 & 3,58 \\
\hline Piptadenia gonoacantha (Mart.) J.F.Macbr. & 4 & 133,3 & 0,42 & 0,001 & 0,001 & 6,67 & 1,22 & 1,65 \\
\hline \multicolumn{9}{|l|}{ Lauraceae } \\
\hline Nectandra nitidula Nees & 1 & 33,3 & 0,11 & 0,0007 & 0,001 & 3,33 & 0,61 & 0,72 \\
\hline \multicolumn{9}{|l|}{ Melastomataceae } \\
\hline Leandra sp. & 533 & $17.766,6$ & 56,58 & 0,002 & 0,002 & 100,00 & 18,29 & 74,88 \\
\hline Miconia corallina Spring & 6 & 200 & 0,64 & 0,12 & 0,11 & 13,33 & 2,44 & 3,18 \\
\hline Miconia latecrenata (DC.) Naudin & 8 & 266,6 & 0,85 & 0,01 & 0,01 & 26,67 & 4,88 & 5,74 \\
\hline Miconia ligustroides (DC.) Naudin & 1 & 33,3 & 0,11 & 0,01 & 0,01 & 3,33 & 0,61 & 0,72 \\
\hline Miconia paulensis Naudin & 2 & 66,6 & 0,21 & 0,69 & 0,64 & 6,67 & 1,22 & 2,07 \\
\hline Miconia tristis Spring & 40 & 1333,3 & 4,25 & 0,001 & 0,001 & 63,33 & 11,59 & 15,83 \\
\hline Miconia urophylla DC. & 4 & 133,3 & 0,42 & 0,01 & 0,01 & 10,00 & 1,83 & 2,26 \\
\hline Tibouchina estrellensis (Raddi) Cogn. & 20 & 666,6 & 2,12 & 0,03 & 0,03 & 43,33 & 7,93 & 10,08 \\
\hline \multicolumn{9}{|l|}{ Myrtaceae } \\
\hline Blepharocalyx salicifolius (Kunth) O.Berg & 1 & 33,3 & 0,11 & 0,85 & 0,78 & 3,33 & 0,61 & 1,50 \\
\hline Marlierea racemosa (Vell.) Kiaersk. & 33 & 1100 & 3,50 & 0,02 & 0,02 & 43,33 & 7,93 & 11,45 \\
\hline Myrcia hebepetala DC. & 28 & 933,3 & 2,97 & 0,02 & 0,02 & 46,67 & 8,54 & 11,53 \\
\hline Myrcia obovata (O.Berg) Nied. & 1 & 33,3 & 0,11 & 0,0004 & 0,0004 & 3,33 & 0,61 & 0,72 \\
\hline \multicolumn{9}{|l|}{ Rubiaceae } \\
\hline Psychotria suterella Müll.Arg. & 1 & 33,3 & 0,11 & 0,04 & 0,04 & 3,33 & 0,61 & 0,75 \\
\hline \multicolumn{9}{|l|}{ Rutaceae } \\
\hline Dictyoloma vandellianum A.Juss. & 4 & 133,3 & 0,42 & 105,63 & 97,64 & 13,33 & 2,44 & 100,51 \\
\hline Citrus limonia Osbeck & 1 & 33,3 & 0,11 & 0,04 & 0,03 & 3,33 & 0,61 & 0,75 \\
\hline \multicolumn{9}{|l|}{ Salicaceae } \\
\hline Xylosma prockia (Turcz.) Turcz. & 1 & 33,3 & 0,11 & 0,01 & 0,01 & 3,33 & 0,61 & 0,73 \\
\hline \multicolumn{9}{|l|}{ Solanaceae } \\
\hline Solanum cinnamomeum Sendtn. & 1 & 33,3 & 0,11 & 0,01 & 0,01 & 3,33 & 0,61 & 0,72 \\
\hline Solanum swartzianum Roem. \& Schult. & 5 & 166,6 & 0,53 & 0,003 & 0,003 & 10,00 & 1,83 & 2,36 \\
\hline \multicolumn{9}{|l|}{ Symplocaceae } \\
\hline Symplocos celastrinea Mart. ex Miq. & 1 & 33,3 & 0,11 & 0,004 & 0,004 & 3,33 & 0,61 & 0,72 \\
\hline Symplocos insignis & 3 & 100 & 0,32 & 0,003 & 0,003 & 6,67 & 1,22 & 1,54 \\
\hline \multicolumn{9}{|l|}{ Theaceae } \\
\hline Laplacea tomentosa (Mart. \& Zucc.) G. Don & 14 & 466,6 & 1,49 & 0,14 & 0,13 & 30,00 & 5,49 & 7,11 \\
\hline \multicolumn{9}{|l|}{ Thymelaeaceae } \\
\hline Daphnopsis brasiliensis Mart. \& Zucc. & 2 & 66,6 & 0,21 & 0,01 & 0,01 & 6,67 & 1,22 & 1,44 \\
\hline Total & 941 & 31.400 & 100 & 108,17 & 100 & 546,67 & 100 & 300 \\
\hline
\end{tabular}


As cinco espécies mais abundantes em 20A corresponderam a $89,4 \%$ dos indivíduos, enquanto em $6 \mathrm{~A}$ o grupo perfez 70,6\%. As espécies mais abundantes em 20A foram Leandra scabra, Bellucia sp., Miconia latecrenata, Psychotria vellosiana e Clethra scabra. Em 6A foram Leandra sp., Croton floribundus, Miconia tristis, Marlierea racemosa e Myrcia hebepetala. Nenhuma espécie se destacou pela abundância em ambas as áreas. As mais abundantes nas duas áreas foram as mesmas que apresentaram os maiores valores de importância (VI), com duas ressalvas: em 20A, Bellucia sp. apresentou o maior VI em razão do maior porte dos indivíduos (e consequente maior dominância), geralmente de caráter cespitosos; em 6A, Dictyoloma vandellianum apresentou o maior VI pelo fato da espécie ter sido representada por arvoretas, resultando em alta dominância. Apenas Miconia latecrenata e Tibouchina estrellensis foram comuns entre as áreas estudadas, o que proporcionou uma baixa similaridade florística (coeficiente de Jaccard de 3,7\%,). Foi verificada, também, significativa diferença entre os índices de diversidade de Shannon $\left(\mathrm{H}^{\prime}\right)(\mathrm{t}=9,63 ; \mathrm{p}<0,001)$, onde $20 \mathrm{~A}\left(\mathrm{H}^{\prime}=2,36\right)$ apresentou maior diversidade que $6 \mathrm{~A}$ $\left(\mathrm{H}^{\prime}=1,55\right)$. A equabilidade de Pielou (J') foi superior na área $20 \mathrm{~A}\left(\mathrm{~J}^{\prime}=0,77\right)$ quando comparada a $6 \mathrm{~A}\left(\mathrm{~J}^{\prime}=0,44\right)$.

As duas áreas possuem solos com textura média (EMBRAPA, 1997) e, de maneira geral, com baixa fertilidade (COMISSÃO DE FERTILIDADE DO SOLODO ESTADO DE MINAS GERAIS - CFSEMG, 1999). Apenas seis parcelas apresentaram textura argilosa (todas em 6A). Houve dissimilaridades edáficas significativas no interior de cada área, sendo que ambas evidenciaram diferenças quanto à acidez trocável $\left(\mathrm{Al}^{3+}\right)$ e potencial $(\mathrm{H}+\mathrm{Al})$ e à capacidade de troca catiônica efetiva $(\mathrm{t})$ e potencial $(\mathrm{T})$. As transeções com maior acidez e CTC foram associadas às populações mais densas e altas de Pteridium (Tabela 2). Entretanto, a maior CTC deve ser analisada de modo multifatorial (CFSEMG, 1999), pois a troca de cátions se associa à fração de argila e aos teores de matéria orgânica (MO) e Al no solo. Desse modo, mesmo apresentando maior CTC, as transeções de elevada dominância de Pteridium apresentaram textura predominantemente arenosa, com baixo teor de $\mathrm{MO}$ e alto $\mathrm{Al}$, o que os torna de baixa fertilidade. O alumínio apresenta maior energia de ligação que os demais cátions, adsorvendo-se às partículas do solo e expondo os cátions à lixiviação (CFSEMG, 1999). Hopkins (1990) aponta que solos de baixa fertilidade e ácidos favorecem o crescimento de Pteridium. Os transectos da área 6A se distinguiram também quanto aos teores de P, K, MO e Premanescente (P-rem). Segundo Hopkins (1990), as populações de Pteridium são beneficiadas em baixos níveis de P disponível, mas em 6A a dominância de Pteridium associou-se aos menores teores de P-rem. Provavelmente, o atributo preponderante para o sucesso da colonização por Pteridium seja a acidez do solo, como observado por Koedam et al. (1992), sendo o restante relacionado, principalmente, à ocupação das áreas pelas demais espécies.

Sabe-se que a serapilheira pode dificultar a regeneração florestal (SANTOS; VÁLIO, 2002). Na área 20A, com maior cobertura e altura de Pteridium (Tabela 2), houve ausência de regenerantes em oito parcelas totalmente cobertas por densas frondes, evidenciando sua atuação como barreira à regeneração. Para Ghorbani et al. (2006), a reabilitação de áreas colonizados por Pteridium é influenciada pela espessura da camada de frondes sobre o solo e suas taxas de deposição e decomposição. Assim, geram tanto limitações físicas à germinação (p.ex. interceptando sementes, que não atingem o solo) como reduzem a radiação nos estratos inferiores (GHORBANI et al., 2006; MATOS; BELINATO, 2010; SILVA; SILVAMATOS, 2006). Isso implica em um modelo de sucessão por inibição (CONNELL; SLATYER, 1977), onde a samambaia atua negativamente sobre a abundância de indivíduos e o número de espécies. Já, em 6A, a cobertura por Pteridium e sua altura foram menores, explicado pelo menor tempo de colonização da área somado ao histórico de menores perturbações sobre o banco de sementes. Ademais, trata-se de terreno menos declivoso, menos favorável ao Pteridium (ALONSO-AMELOT et al., 2004, 2007). De acordo com Ghorbani et al. (2006), diferenças estruturais, de composição e de diversidade na vegetação podem surgir, em decorrência do Pteridium. E há os efeitos alelopáticos relacionados à espécie, que asseguram a sua dominância (GLIESSMAN, 1976; GLIESSMAN; MULLER, 1972; MATOS; PIVELLO, 2009; SAITO et al., 1989). Assim, as menores abundância e riqueza em 20A resultam da interação negativa Pteridium-flora nativa, já que a área é circundada por florestas que atuam como fonte de propágulos.

A maior diversidade em 20A não deve ser compreendida como indicador da recuperação da vegetação, pois o desequilíbrio competitivo a favor do Pteridium torna regular a distribuição de indivíduos das demais espécies, reduzindo suas abundâncias e interferindo no cálculo da diversidade que (GUREVITCH et al., 2009) é proporcional à riqueza e também à equidade de abundâncias entre populações.

Cerne, Lavras, v. 19, n. 1, p. 65-76, jan./mar. 2013 
Tabela 2 - Variáveis edáficas e topográficas, e parâmetros de cobertura e altura média de Pteridium aquilinum obtidos em um inventário da regeneração natural em dois diferentes trechos localizados em Bocaina de Minas, MG. ${ }^{+}$, não foi realizado cálculo do desvio padrão; * e ${ }^{+}$, parâmetros que apresentaram diferenças estatísticas significativas nas áreas 20A e 6A, respectivamente; Diferentes letras correspondem à significância estatística $(\alpha<0,05)$.

Table 2 - Edaphic and topographic variables and Pteridium aquilinum coverage and average height parameters obtained in the inventory of natural regeneration in two different areas in Bocaina de Minas, $M G^{+}{ }^{+}$, standard deviation was not calculated; * and ${ }^{+}$, parameters that showed statistically significant differences in the $20 \mathrm{~A}$ and $6 \mathrm{~A}$ areas, respectively. Different letters correspond to statistical significance $(\alpha<0.05)$.

\begin{tabular}{|c|c|c|c|c|c|}
\hline \multirow{2}{*}{ Variáveis } & \multicolumn{3}{|c|}{ Área 20A } & \multicolumn{2}{|c|}{ Área 6A } \\
\hline & Trans. 1 & Trans. 2 & Trans. 1 & Trans. 2 & Trans. 3 \\
\hline \multicolumn{6}{|l|}{ Químicas } \\
\hline $\mathrm{pH} \mathrm{em} \mathrm{H}_{2} \mathrm{O}$ & $4,99 \pm 0,14$ & $4,87 \pm 0,17$ & $4,69 \pm 0,17$ & $4,56 \pm 0,10$ & $4,56 \pm 0,22$ \\
\hline P-Mehlich $\left(\mathrm{mg}^{\left.-\mathrm{dm}^{-3}\right)^{+}}\right.$ & $3,99 \pm 1,17$ & $4,06 \pm 1,17$ & $1,5 \pm 0,31^{\mathrm{a}}$ & $1,13 \pm 0,18^{\mathrm{b}}$ & $1,1 \pm 0,15^{\mathrm{b}}$ \\
\hline $\mathrm{K}^{+}\left(\mathrm{mg} \cdot \mathrm{dm}^{-3}\right)^{+}$ & $78,5 \pm 45,6$ & $82,2 \pm 40,8$ & $25,1 \pm 5,9^{\mathrm{a}}$ & $21 \pm 6,8^{\mathrm{ab}}$ & $16,6 \pm 4,7^{\mathrm{b}}$ \\
\hline $\mathrm{Ca}^{2^{+}}\left(\mathrm{cmolc} \mathrm{cm}^{-3}\right)$ & $0,14 \pm 0,05$ & $0,20 \pm 0,13$ & $0,1 \pm 0,00$ & $0,1 \pm 0,00$ & $0,11 \pm 0,04$ \\
\hline $\mathrm{Mg}^{2^{+}}\left(\mathrm{cmolc} \mathrm{cm}^{-3}\right)$ & $0,12 \pm 0,07$ & $0,12 \pm 0,04$ & $0,1 \pm 0,00$ & $0,1 \pm 0,00$ & $0,1 \pm 0,00$ \\
\hline $\mathrm{Al}^{3+}\left(\mathrm{cmolc} \mathrm{cm}^{-3}\right)^{*+}$ & $1,86 \pm 0,19$ & $2,20 \pm 0,37$ & $1,28 \pm 0,15^{\mathrm{a}}$ & $1,18 \pm 0,09^{\mathrm{a}}$ & $0,95 \pm 0,21^{\mathrm{b}}$ \\
\hline $\mathrm{H}+\mathrm{Al}\left(\mathrm{cmolc}^{\mathrm{cm}} \mathrm{cm}^{-3}\right) *+$ & $13,2 \pm 1,42$ & $15,1 \pm 2,23$ & $10,2 \pm 1,36^{\mathrm{a}}$ & $8,3 \pm 0,73^{\mathrm{b}}$ & $7,6 \pm 1,41^{\mathrm{b}}$ \\
\hline 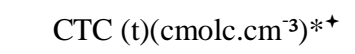 & $2,34 \pm 0,3$ & $2,74 \pm 0,4$ & $1,57 \pm 0,16^{\mathrm{a}}$ & $1,45 \pm 0,11^{\mathrm{a}}$ & $1,2 \pm 0,21^{\mathrm{b}}$ \\
\hline CTC $(\mathrm{T})\left(\mathrm{cmolc} \mathrm{cm}^{-3}\right)^{*^{+}}$ & $13,6 \pm 1,4$ & $15,6 \pm 2,2$ & $10,4 \pm 1,37^{\mathrm{a}}$ & $8,6 \pm 0,74^{\mathrm{b}}$ & $7,9 \pm 1,42^{\mathrm{b}}$ \\
\hline $\mathrm{SB}\left(\mathrm{cmolc} \cdot \mathrm{cm}^{-3}\right)$ & $0,47 \pm 0,2$ & $0.54 \pm 0,24$ & $0.29 \pm 0,02$ & $0,27 \pm 0,04$ & $0,25 \pm 0,05$ \\
\hline $\mathrm{MO}\left(\text { dag. } \mathrm{kg}^{-1}\right)^{+}$ & $5,90 \pm 0,85$ & $5,64 \pm 0,82$ & $3,87 \pm 0,59^{\mathrm{a}}$ & $2,83 \pm 0,17^{\mathrm{b}}$ & $2,5 \pm 0,36^{\mathrm{b}}$ \\
\hline P-rem $\left(\mathrm{mg} . \mathrm{L}^{-1}\right)^{+}$ & $4,56 \pm 2,2$ & $4,03 \pm 1,6$ & $7,8 \pm 1.3^{\mathrm{a}}$ & $10,3 \pm 2,9^{\mathrm{b}}$ & $14,5 \pm 1,9^{\mathrm{c}}$ \\
\hline $\mathrm{V}(\%)^{+}$ & 3,5 & 3,23 & 2,6 & 3 & 3,38 \\
\hline $\mathrm{m}(\%)^{+}$ & 80,2 & 81,7 & 82,6 & 82,2 & 77,8 \\
\hline \multicolumn{6}{|l|}{ Granulométricas } \\
\hline Areia $\left(\text { dag. } \mathrm{kg}^{-1}\right)^{+}$ & 59,2 & 55,9 & 51,8 & 51,2 & 48,3 \\
\hline Silte (dag.kg-1) ${ }^{+}$ & 17,1 & 20,2 & 15 & 16,6 & 16,2 \\
\hline Argila (dag.kg $\left.{ }^{-1}\right)^{+}$ & 23,8 & 23,9 & 33,3 & 32,3 & 35,5 \\
\hline \multicolumn{6}{|l|}{ Topográficas } \\
\hline Desnível (m) & $39,2 \pm 25,1$ & $31,1 \pm 18,5$ & $25,2 \pm 17,5$ & $24,2 \pm 15,9$ & $12,2 \pm 8,3$ \\
\hline Altitude (m) & $1.324,2 \pm 25,1$ & $1.315,5 \pm 18,2$ & $1.194,2 \pm 17,5$ & $1.192 .9 \pm 15,9$ & $1.180,5 \pm 8,3$ \\
\hline \multicolumn{6}{|l|}{ Presença de Pteridium } \\
\hline Altura $(\mathrm{m}) *^{+}$ & $2,32 \pm 0,7$ & $3,43 \pm 0,5$ & $1,58 \pm 0,3^{\mathrm{a}}$ & $1,54 \pm 0,4^{\mathrm{a}}$ & $1 \pm 0,3^{\mathrm{b}}$ \\
\hline Cobertura $(\%)^{+}$ & 81,4 & 96,7 & 56,3 & 68,8 & 46,7 \\
\hline
\end{tabular}

Já, 6A apresentou maior riqueza de espécies, mas baixa equabilidade, indicando a concentração de abundância em poucas espécies, principalmente em Leandra sp. e Croton floribundus. A importância dessas espécies pode decorrer da capacidade de rebrota observada em 6A (mas não quantificada). A regeneração por gemas fisiologicamente ativas em locais perturbados é frequente e essencial, quando as condições para as sementes são limitadas (VESK; WESTOBY, 2004). Assim, a rebrota permite rápida recolonização com menor efeito negativo sobre a riqueza, o que pode explicar o maior número de indivíduos e espécies regenerantes em $6 \mathrm{~A}$, sugerindo a necessidade de pesquisas 
que considerem tal estratégia. No entanto, ainda que as populações de Pteridium interfiram na regeneração florestal, os distintos históricos de perturbação entre áreas devem ser ponderados. Conforme a conjugação de atributos como tipo, frequência e intensidade de distúrbios, há modificações na dinâmica da vegetação (CONNELL, 1978). Em 20A, a introdução da espécie Melinis minutiflora para formação de pastagem pode ter eliminado os propágulos de espécies nativas, retardando a regeneração. Sabe-se que a formação de pastagens promove alterações ambientais (GÓMEZ-POMPA; VÁZQUES-YANES, 1981; HORN, 1980; PARROTTA et al., 1997), com mudanças físicas e químicas do solo(GUGGENBERGER; ZECH, 1999).

$\mathrm{Na}$ análise de correspondência canônica, houve nítida separação quanto à composição de espécies entre as duas áreas (Figura 1). Verificou-se colinearidade entre as variáveis $\mathrm{H}+\mathrm{Al}$ e $\mathrm{Al}, \mathrm{t}$ e T, além da complementariedade que existe entre areia, silte e argila. Desse modo, foram retiradas da análise as variáveis $\mathrm{H}+\mathrm{Al}, \mathrm{T}$ e silte por interferirem na independência entre as variáveis, ou seja, por reduzir a ortogonalidade entre os eixos (HAIR et al., 2009). Apenas Mg, saturação por bases (V) e por Al (m) apresentaram baixas correlações com os eixos de ordenação, com consequente baixa explicação sobre o gradiente de ordenação $\left(\mathrm{r}^{2}<0,3\right)$ (BRAAK, 1987). O primeiro eixo da CCA apresentou elevado autovalor (Tabela 3), indicando alta substituição de espécies entre as áreas. Os dois primeiros eixos explicaram 19,5\% da variância global, com elevadas correlações entre as comunidades inventariadas e as variáveis edáficas, topográficas e da população de Pteridium. A alta variância remanescente não explicada é comum em pesquisas de vegetação e não interfere na significância entre as relações espécie-ambiente (BRAAK, 1987; BRAAK; PRENTICE, 1988).

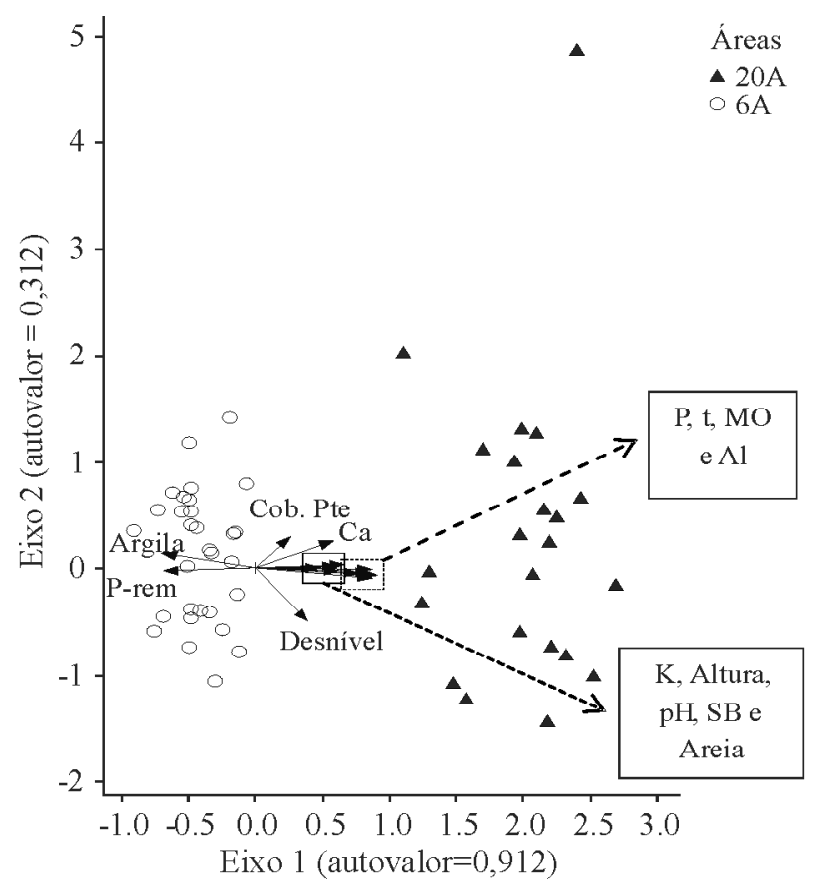

Figura 1 - Ordenação das parcelas segundo as relações entre espécies e variáveis de solo, topográfica e da população de Pteridium após a análise de correspondência canônica (CCA), obtida para as comunidades de espécies arbustivas e arbóreas presentes na regeneração natural em duas áreas colonizadas por Pteridium aquilinum, localizadas em Bocaina de Minas, MG. As variáveis no interior dos quadros estão organizadas em ordem decrescente quanto a correlação com os eixos de ordenação.

Figure 1 - Plots ordination according to the relation between species and soil, topographic and Pteridium population variables after a canonical correspondence analysis (CCA), obtained for the shrub and tree communities present in the natural regeneration in two areas occupied by Pteridium aquilinum, and located in Bocaina de Minas, MG. The variables inside the frames were arranged in descending order of correlation with the ordination axes.

Cerne, Lavras, v. 19, n. 1, p. 65-76, jan./mar. 2013 
Tabela 3 - Resumo dos resultados da análise de correspondência canônica (CCA) para espécies arbustivas e arbóreas regenerantes em Bocaina de Minas, MG.

Table 3 - Summary of the data from canonical correspondence analysis (CCA) for the natural regeneration of shrub and tree species in Bocaina de Minas, $M G$.

\begin{tabular}{lccc}
\hline & Eixo 1 & Eixo 2 & Eixo 3 \\
\hline Autovalores & 0,912 & 0,312 & 0,304 \\
Variância os dados das espécies (\%) & 14,5 & 5 & 4,9 \\
Variância explicada cumulativa (\%) & 14,6 & 19,5 & 24,4 \\
Correlações espécie-ambiente & 0,98 & 0,814 & 0,826 \\
Significância das correlações pelo teste Monte Carlo. & 0,001 & 0,001 & 0,001 \\
\hline
\end{tabular}

Os principais atributos responsáveis pela formação do grupo de parcelas de 6A foram o solo argiloso e maiores teores de P-rem (Figura 1). Já, os maiores teores de K, P, $\mathrm{Ca}, \mathrm{Al}$ e MO, e maiores CTC, soma de bases trocáveis (SB) e pH ocasionaram o agrupamento das parcelas de $20 \mathrm{~A}$, assim como o desnível mais acentuado e maiores cobertura e altura de Pteridium (forte e positivamente correlacionadas com o eixo 1). A maior disponibilidade de nutrientes pode decorrer de sua ciclagem a partir das frondes. Por outro lado, o relevo pode ter influenciado o teor de Al, já que áreas mais íngremes (como em 20A) sofrem maior lixiviação de nutrientes, aumentando a acidez do solo (RODRIGUES et al., 2003). A maior altitude em 20A, mais dominada por Pteridium, concorda com Alonso-Amelot et al. (2007), para quem os ambientes mais elevados favorecem as populações desta samambaia. E tal contexto reforça as afirmações de Hamilton e McMillan (2004) sobre a fragilidade ambiental dos ambientes montanos.

Diante da dominância de Pteridium aquilinum em áreas florestais perturbadas, Matos e Belinato (2010) sugeriram o desbaste da samambaia como forma de reabilitação ambiental. Essa pode ser uma estratégia viável em áreas como 20A, que mesmo após 20 anos em regeneração natural não foi capaz de restaurar sua fisionomia ou estrutura florestal. Também é recomendável o manejo de Pteridium em 6A, pois a espécie produz substâncias potencialmente carcinogênicas (GHORBANI et al., 2006) e a área encontra-se próxima ao manancial de água, que pode ser contaminado (embora haja carência de informações sobre a dinâmica dessas substâncias no ambiente). Importante observar também que as populações de Pteridium favorecem a ocorrência de incêndios pelo acúmulo de matéria seca, principalmente frondes (MIATTO et al., 2011; SILVA; SILVA-MATO, 2006), o que promove sua expansão.

\section{CONCLUSÕES}

A dominância de Pteridium aquilinum em áreas montanas desflorestadas na Serra da Mantiqueira conduz a uma sucessão ecológica sob inibição, onde a samambaia atua negativamente sobre a riqueza e abundância de populações arbustivo-arbóreas. É confirmada a preferência da espécie por terrenos mais declivosos, a maiores altitudes, com solos de elevado teor de alumínio e, consequentemente, mais ácidos, beneficiando-se também de distúrbios. Ressalta-se a suscetibilidade desses ambientes montanos e a importância da preservação de sua vegetação natural, assim como do desbaste das samambaias como estratégia de restauração florestal.

\section{REFERÊNCIAS}

ALONSO-AMELOT, M. E.; OLIVEROS, A. B.;

CALCAGNO-PISARELLI, M. P. Phenolics and condensed tannins in relation to altitude in neotropical Pteridium spp. a field study in the Venezuelan Andes. Biochemical

Systematics and Ecology, Oxford, v. 32, n. 11, p. 969-981, Nov. 2004.

ALONSO-AMELOT, M. E.; OLIVEROS, A. B.; CALCAGNO-PISARELLI, M. P. Phenolics and condensed tannins of high altitude Pteridium arachnoideum in relation to sunlight exposure, elevation, and rain regime. Biochemical Systematics and Ecology, Oxford, v. 35, n. 1, p. 1-10, Jan. 2007.

ANGIOSPERM PHYLOGENY GROUP. An update of the Angiosperm Phylogeny Group classification for the orders and families of flowering plants: APG II. Botanical Journal of the Linnean Society, London, v. 141, n. 4, p. 399-436, Apr. 2003. 
AYRES, J. M. Os corredores ecológicos das florestas tropicais do Brasil. Belém: Sociedade Civil Mamirauá, 2005. $256 \mathrm{p}$.

BRAAK, C. J. F. ter. The analysis of vegetation environment relathionships by canonical correspondence analysis. Vegetatio, Dordrecht, v. 63, n. 3, p. 69-77, May/ June 1987.

BRAAK, C. J. F. ter; PRENTICE, I. C. A theory of gradiente analysis. Advances in Ecological Research, London, v. 18, n. 2, p. 271-317, 1988.

BRAUN-BLANQUET, J. Fitosociología: bases para yo estúdio de las comunidades vegetales. Madrid: Blume, 1979. $820 \mathrm{p}$.

BROKAW, N. V. L. The definition of treefall gap and its effect on measures of forest dynamics. Biotropica, Lawrense, v. 14, n. 2, p. 158-160, June 1982.

CAPOBIANCO, J. P. R. (Org.). Dossiê Mata Atlântica. Brasília: RMA, 2001. 407 p.

CARVALHO, D. A.; OLIVEIRA-FILHO, A. T.; BERG, E. V. D.; FONTES, M. A. L.; VILELA, E. A.; MARQUES, J. J. G. S. M.; CARVALHO, W. A. C. Variações florísticas e estruturais do compartimento arbóreo de uma floresta ombrófila alto-montana às margens do Rio Grande, Bocaina de Minas, MG, Brasil. Acta Botânica Brasílica, Porto Alegre, v. 19, n. 1, p. 91-109, jan./mar. 2005.

COMISSÃO DE FERTILIDADE DO SOLO DO ESTADO DE MINAS GERAIS. Recomendações para o uso de corretivos e fertilizantes em Minas Gerais. 5. ed. Lavras, 1999. 359 p.

CONNELL, J. H. Diversity in tropical rainforest and coral reefs. Science, Washington, v. 199, n. 4335, p. 1302-1310, Mar. 1978.

CONNELL, J. H.; SLATYER, R. O. Mechanisms of sucession in natural communities and their role in community stability and organization. The American Naturalist, Chicago, v. 111, n. 982, p. 1119-1144, Nov./Dec. 1977.

DENSLOW, J. S. Gap partitioning among tropical rainforest trees. Biotropica, Lawrence, v. 12, n. 2, p. 47-55, June 1980.
EMPRESA BRASILEIRA DE PESQUISA

AGROPECUÁRIA. Centro Nacional de Pesquisa de Solos. Manual de métodos de análise de solo. Rio de Janeiro, 1997. $212 \mathrm{p}$.

\section{EMPRESA BRASILEIRA DE PESQUISA}

AGROPECUÁRIA. Centro Nacional de Pesquisa de Solos. Sistema brasileiro de classificação de solos. Rio de Janeiro, 2006. 306 p.

FRELICH, L. E.; SUGITA, S.; REICH, P. B.; DAVIS, M. B.; FRIEDMAN, S. K. Neighbourhood effects in forests: implications for within-stand patch structure. Journal of Ecology, London, v. 86, n. 1, p. 149-161, Jan. 1998.

\section{FUNDAÇÃO SOS MATA ATLÂNTICA; INSTITUTO} NACIONAL DE PESQUISAS ESPACIAIS. Atlas dos remanescentes florestais da mata atlântica: período 20052008: relatório parcial. São Paulo, 2009. 156 p.

GHORBANI, J. A.; LE DUC, M. G.; MCALLISTER, H. A. Effects of the litter layer of Pteridium aquilinum on seed banks under experimental restoration. Applied Vegetation Science, New York, v. 9, n. 1, p. 127-136, May 2006.

GLIESSMAN, S. R. Allelopathy in a broad spectrum of environments as illustrated by bracken. Botanical Journal of the Linnean Society, London, v. 73, n. 1, p. 95-104, Apr. 1976.

GLIESSMAN, S. R.; MULLER, C. H. The phytotoxic potential of bracken, Pteridium aquilinum (L.) Kuhn. Madrono: West American Journal of Botany, Berkeley, v. 21, n. 1, p. 299-304, Jan./Mar. 1972.

GOMEZ-POMPA, A.; VÁZQUEZ-YANES, C. Sucessional studies of a rain forest in Mexico. In: WEST, D. C.; SHUGAR, H. H.; BOTKIN, D. B. (Ed.). Forest sucession: concepts and aplications. New York: Spriger-Verlag, 1981.p. 246-266.

GUGGENBERG, G.; ZECH, W. Soil organic matter composition under primary forest, pasture and secondary forest sucession, Región Huetar Norte, Costa Rica. Forest Ecology and Management, Amsterdam, v. 124, n. 1, p. 96104, Jan. 1999.

GUREVITCH, J.; SCHEINER, S. M.; FOX, G. A. Ecologia vegetal. São Paulo: Artmed, 2009. 592 p.

Cerne, Lavras, v. 19, n. 1, p. 65-76, jan./mar. 2013 
HAIR JÚNIOR, J. F.; BLACK, W. C.; BABIN, B. J.; ANDERSON, R. E.; TATHAN, R. L. Análise

multivariada de dados. Porto Alegre: Bookman, 2009. 688 p.

HAMILTON, L.; MCMILLAN, L. Guidelines for planning and managing mountain protected areas. Cambridge: IUCN, 2004. 83 p.

HONDA, Y. Ecological correlations between the persistence of the soil seed bank and several plant traits, including seed dormancy. Plant Ecology, Heidelberg, v. 196, n. 2, p. 301309, June 2008.

HOPKINS, N. C. G. Bracken (Pteridium aquilinum): its distribuition and animal health implications. British Veterinary Journal, London, v. 146, n. 4, p. 316-326, Apr. 1990.

HORN, H. S. Some causes of variety in patterns of secondary sucession. In: WEST, D. C.; SHUGAR, H. H.; BOTKIN, D. B. (Ed.). Forest sucession: concepts and aplications. New York: Spriger-Verlag, 1980. p. 25-55.

JOLY, A. B. Botânica: introdução à taxonomia vegetal. São Paulo: Nacional, 1998. 778 p.

KOEDAM, N.; BÜSCHER, P.; SPEYBROECK, D. van. Acidiphily in pteridophytes: assessment of the role of root cation-exchange properties. Journal of Plant Nutrition, Windsor, v. 15, n. 12, p. 2605-2619, 1992.

KREBS, C. J. Ecological methodologiy. New York: Harper \& Row, 1989. 654 p.

MATOS, D. M. S.; BELINATO, T. A. Interference of Pteridium arachnoideum (Kaulf.) Maxon. (Dennstaedtiaceae) on the establishment of rainforest trees. Brazilian Journal of Biology, São Carlos, v. 70, n. 2, p. 311-316, maio 2010.

MATOS, D. M. S.; PIVELLO, V. R. O impacto das plantas invasoras nos recursos naturais de ambientes terrestres: alguns casos brasileiros. Ciência e Cultura, São Paulo, v. 61, n. 1, p. 27-30, jan. 2009.

MCCUNE, B.; MEFFORD, M. J. PC-ORD: multivariate analysis of ecological data. Version 5.10. Oregon: Gleneden Beach, 2006. 126 p.
MIATTO, R. C.; SILVA, I. A.; MATOS, D. M. S.; MARRS, R. $\mathrm{H}$. Woody vegetation structure of Brazilian Cerrado invaded by Pteridium arachnoideum (Kaulf.) Maxon (Dennstaedtiaceae).

Flora, London, v. 206, n. 8, p. 757-762, Aug. 2011.

MUELLER-DOMBOIS, D.; ELLENBERG, H. Aims and methods of vegetation ecology. New York: J. Wiley, 1974. 547 p.

OLIVEIRA-FILHO, A. T.; CARVALHO, D. A.; FONTES, M. A. L.; BERG, E. van den; CURI, N.; CARVALHO, W. A. C. Variações estruturais do compartimento arbóreo de uma floresta semidecídua alto-montana na chapada das Perdizes, Carrancas, MG. Revista Brasileira de Botânica, São Paulo, v. 27, n. 2, p. 291-309, abr./jun. 2004.

OLIVEIRA FILHO, A. T.; FONTES, M. A. L. Patterns of floristic differentiation among Atlantic forests in Southeastern Brazil and the influence of climate. Biotropica, Lawrence, v. 32, n. 4, p. 793-810, 2000.

OLIVEIRA-FILHO, A. T.; MELLO, J. M. de; SCOLFORO, F. R. Effects of past disturbance and edges on tree community struture ande dynamics withim a fragment of tropical semideciduos Forest in south-eastern Brazil over a Five year period: 1987 - 1992. Plant Ecology, Dordrecht, v. 131, n. 1, p. 45-66, Jan./Feb. 1997.

\section{PARROTTA, J. A.; KNOWLES, O. H.; WUNDERLE} JÚNIOR, J. M. Development of floristic diversity in 10-yearold restoration forests on a bauxite mined site in Amazonia. Forest Ecology and Management, Amsterdam, v. 99, n. 1, p. 21-42, Jan. 1997.

PINHEIRO, E. S.; DURIGAN, G. Dinâmica espaço-temporal (1962-2006) das fitofisionomias em unidade de conservação do Cerrado no sudeste do Brasil. Revista Brasileira de Botânica, São Paulo, v. 32, n. 3, p. 441-454, jul./set. 2009.

PINTO, L. V. A.; BOTELHO, S. A.; OLIVEIRA-FILHO, A. T.; DAVIDE, A. C. Estudo da vegetação como subsídios para proposta de recuperação de nascentes da Bacia Hidrográfica do Ribeirão Santa Cruz, Lavras, MG. Revista Árvore, Viçosa, v. 29, n. 5, p. 775-793, set./out. 2005.

RODRIGUES, L. A.; CARVALHO, D. A.; OLIVEIRAFILHO, A. T.; BOTREL, R. T.; SILVA, E. A. Florística e estrutura da comunidade arbórea de um fragmento florestal em Luminárias, MG. Acta Botanica Brasílica, São Paulo, v. 17, n. 1 , p. $71-87$, jan./mar. 2003.

Cerne, Lavras, v. 19, n. 1, p. 65-76, jan./mar. 2013 
RODRIGUES, R. R.; TORRES, R. B.; MATHES, L. A.; PENHA, A. S. Tree species sprouting from root buds in a semideciduous forest affected by fires. Brazilian Archives of Biology and Technology, Curitiba, v. 47, n. 1, p. 127-133, Mar. 2004.

RONDON NETO, R. M.; BOTELHO, S. A.; FONTES, M. A. L.; DAVIDE, A. C.; FARIA, J. M. R. Estrutura e composição florística da comunidade arbustivo-arbórea de uma clareira de origem antrópica, em uma floresta estacional semidecídua Montana, Lavras, MG, Brasil. Cerne, Lavras, v. 6, n. 2, p. 79-94, 2000.

SAITO, K.; NAGAO, T.; MATOBA, M.; KOYAMA, K.; NATORI, S.; MURAKAMI, T.; SAIKI, Y. Chemical assay of ptaquiloside, the carcinogen of Pteridium aquilinum, and the distribution of related compounds in the Pteridaceae. Phytochemistry: Chemistry, Biochemistry, Molecular Biology, New York, v. 28, n. 6, p. 1605-1611, June 1989.

SANTOS, S. L.; VÁLIO, I. F. M. Litter accumulation and its effect on seedling recruitment in a Southeast Brazilian Tropical Forest. Revista Brasileira de Botânica, São Paulo, v. 25, n. 1, p. 89-92, mar. 2002.
SILVA, U. S. R.; SILVA-MATOS, D. M. The invasion of Pteridium aquilinum and the impoverishment of the seed bank in fire prone areas of Brazilian Atlantic Forest. Biodiversity and Conservation, London, v. 15, n. 9, p. 3035-3043, Aug. 2006.

SOUZA, A. L.; SCHETTINO, S.; JESUS, R. M.; VALE, A. B. Dinâmica da regeneração natural em uma floresta ombrófila densa secundária, após corte de cipós, Reserva Natural da Companhia Vale do Rio Doce S. A. Estado Espírito Santo, Brasil. Revista Árvore, Viçosa, v. 26, n. 4, p. 411-419, jul./ ago. 2002.

UHL, C.; BUSCHBACKER, R.; SERRÃO, E. A. S. Abandoned pastures in eastern Amazonia: I., patterns of plant sucession. Journal of Ecology, Oxford, v. 76, n. 2, p. 663681, May/Aug. 1988.

VESK, P. A.; WESTOBY, M. Sprouting ability across diverse disturbance and vegetation types worldwild. Journal of Ecology, London, v. 92, n. 2, p. 310-320, Feb. 2004.

ZAR, J. H. Biostatistical analysis. New Jersey: PrenticeHall, 1996. 718 p. 